\title{
Quasi-periodic oscillations: Resonant shear Alfvén waves in neutron star magnetospheres
}

\author{
V. Rezania ${ }^{1,2}$ and J. C. Samson ${ }^{1}$ \\ 1 Theoretical Physics Institute, Department of Physics, University of Alberta, Edmonton, AB, T6G 2J1^, Canada \\ e-mail: vrezania@phys.ualberta.ca \\ 2 Institute for Advanced Studies in Basic Sciences, Zanjan 45195, Iran
}

Received 5 August 2004 / Accepted 19 February 2005

\begin{abstract}
We propose a new model for quasi-periodic oscillations (QPOs) based on oscillating magnetohydrodynamic modes in neutron star magnetospheres. We argue that the interaction of the accretion disk with the magnetosphere can excite resonant shear Alfvén waves in a region of enhanced density gradients, the region where accretion material flows along the magnetic field lines in the magnetosphere (see Fig. 1). We demonstrate that depending on the distance of this region from the star and the magnetic field strength, the frequency of the field line resonance can range from several $\mathrm{Hz}$ (a weaker field, farther from the star), to approximately $\mathrm{kHz}$ frequencies (a stronger field, $\sim 2-10$ stellar radii from the star). We show that such oscillations are able to significantly modulate inflow of matter from the high density region toward the star's surface and possibly produce the observed X-ray spectrum. In addition, we show that the observed 2:3 frequency ratio of QPOs is a natural result of our model.
\end{abstract}

Key words. stars: neutron - stars: magnetic fields - X-rays: binaries

\section{Introduction}

The discovery of quasi-periodic oscillations (QPOs) in lowmass X-ray binaries (LMXBs) has been reported and discussed in recent studies in theoretical and observational astrophysics. The Rossi X-Ray Timing Explorer has observed oscillations in the X-ray flux of about 20 accreting neutron stars. These oscillations are very strong and remarkably coherent with frequencies ranging from $\sim 10 \mathrm{~Hz}$ to $\sim 1200 \mathrm{~Hz}$. The $\mathrm{kHz}$ frequencies correspond to dynamical time scales at radii of a few tens of kilometers, and are possibly closely related to the Keplerian orbital frequency of matter in the inner disk. Almost all sources have also shown twin spectral peak QPOs in the $500 \mathrm{~Hz}$ to $1200 \mathrm{~Hz}$ part of the X-ray spectrum, and both peaks move up and down in frequency simultaneously.

Although there are some detailed differences between different types of X-ray sources, the observed QPOs are remarkably similar, both in frequency and peak separation. (Six of the 20 known sources were originally identified as "Z" sources and the rest are known as "atoll" sources (see Table 1). For more information about the atoll and Z sources, see van der Klis 2000). Such a similarity shows that QPOs should depend on the general characteristics of the X-ray sources which are common to all systems. In other words, the QPO can be regarded as a generic feature of the accreting neutron star.

\footnotetext{
^ Present address.
}

Remarkably, the characteristic dynamical time scale for material orbiting near the compact object is comparable to the observed millisecond X-ray variability, i.e. because $\tau_{\mathrm{dyn}}=$ $\sqrt{r^{3} /(G M)} \sim 2 \mathrm{~ms}(r / 100 \mathrm{~km})^{3 / 2}\left(1.4 M_{\odot} / M\right)^{1 / 2}$ where $r$ is the distance of the orbiting material from the center of the compact object with mass $M$ (circular orbit), $G=6.67 \times$ $10^{-8} \mathrm{dyn} \mathrm{cm}^{2} / \mathrm{g}^{2}$, and $M_{\odot}=1.99 \times 10^{33} \mathrm{~g}$. Such a natural time scale is the foundation of most models for the observed QPOs. Alpar \& Shaham (1985) first proposed a beat-frequency model to explain the low-frequency ${ }^{1}$ horizontal branch oscillation (HBO) seen in Z sources (see also Lamb et al. 1985). They used the Alfvén radius, where the magnetic pressure balances the ram pressure of the infalling material in spherical accretion, $r_{\mathrm{A}} \sim 1.5 \times 10^{6} \mathrm{~cm} \dot{M}_{17}^{-2 / 7} \mu_{26}^{4 / 7}\left(M / M_{\odot}\right)^{-1 / 7}$, as the preferred radius. For this Alfvén radius, the assumptions are: the magnetic field is stellar dipole, the velocity and mass density are for the free-fall case. Here $\dot{M}_{17}$ is the mass accretion rate (this corresponds to $1.5 \times 10^{-9} M_{\odot} \mathrm{yr}^{-1}$ and is typical of low-mass accreting neutron stars) in units of $10^{17} \mathrm{~g} \mathrm{~s}^{-1}$ and $\mu_{26}$ is the magnetic dipole moment at the stellar surface in units of $10^{26} \mathrm{G} \mathrm{cm}^{3}$. Note that we assumed that $\dot{M}$ is constant in time and position.

Miller et al. $(1996,1998)$ proposed a beat-frequency model to study $\mathrm{kHz} \mathrm{QPOs}$, the so-called sonic-point beat-frequency

\footnotetext{
1 Two different low-frequency $(<100 \mathrm{~Hz})$ QPOs were known in the $\mathrm{Z}$ sources, the $6-20 \mathrm{~Hz}$ so-called normal and flaring-branch oscillation (NBO; Middleditch \& Priedhorsky 1986) and the 15-60 Hz so-called horizontal branch oscillation (HBO; van der Klis et al. 1985).
} 
Table 1. Observed frequencies of kilohertz QPOs in Z and atoll sources.

\begin{tabular}{lccclcc}
\hline \hline & & $v_{1}$ & $v_{2}$ & $\Delta v$ & $M_{\mathrm{s}} / R_{\mathrm{s}}^{3}$ & $\begin{array}{c}\mu_{\mathrm{s}} \\
\left(10^{14} \mathrm{~g} \mathrm{~cm}^{-3}\right)\end{array}$ \\
Source & Type & $(\mathrm{Hz})$ & $(\mathrm{Hz})$ & $(\mathrm{Hz})$ & $\left.\mathrm{G} \mathrm{cm}^{3}\right)$ \\
\hline Sco X-1 & $\mathrm{Z}$ & 565 & 870 & $307 \pm 5$ & 2.6 & 6.6 \\
GX 5-1 & $\mathrm{Z}$ & 660 & 890 & $298 \pm 11$ & 2.7 & 7.6 \\
GX 17+2 & $\mathrm{Z}$ & 780 & 1080 & $294 \pm 8$ & 3.6 & 8.4 \\
Cyg X-2 & $\mathrm{Z}$ & 660 & 1005 & $346 \pm 29$ & 3.5 & 7.2 \\
GX 340+0 & $\mathrm{Z}$ & 565 & 840 & $339 \pm 8$ & 2.6 & 6.6 \\
GX 349+2 & $\mathrm{Z}$ & 710 & 980 & $266 \pm 13$ & 2.9 & 8.0 \\
4U 0614+09 & atoll & 825 & 1160 & $312 \pm 2$ & 4.1 & 8.6 \\
4U 1608-52 & atoll & 865 & 1090 & $225 \pm 12$ & 3.3 & 9.5 \\
4U 1636-53 & atoll & 950 & 1190 & $251 \pm 4$ & 3.9 & 10.0 \\
4U 1702-43 & atoll & 770 & 1085 & $315 \pm 11$ & 3.7 & 8.2 \\
4U 1705-44 & atoll & 775 & 1075 & $298 \pm 11$ & 3.6 & 8.3 \\
4U 1728-34 & atoll & 875 & 1160 & $349 \pm 2$ & 4.3 & 9.0 \\
KS 1731-260 & atoll & 900 & 1160 & $260 \pm 10$ & 3.8 & 9.5 \\
4U 1735-44 & atoll & 900 & 1150 & $249 \pm 15$ & 3.7 & 9.6 \\
4U 1820-30 & atoll & 795 & 1075 & $278 \pm 11$ & 3.5 & 8.6 \\
Aq1 X-1 & atoll & 930 & 1040 & $241 \pm 9$ & 3.1 & 10.3 \\
4U 1915-05 & atoll & 655 & 1005 & $348 \pm 11$ & 3.5 & 7.2 \\
XTE J2123-058 & atoll & 845 & 1100 & $255 \pm 14$ & 3.5 & 9.2 \\
\hline
\end{tabular}

Low $\left(v_{1}\right)$ and high $\left(v_{2}\right)$ QPO frequencies with corresponding peak separation $(\Delta v)$ observed in $\mathrm{Z}$ and atoll sources. Only one data series is given as an example; for further detail see van der Klis (2000). Values of $M_{\mathrm{s}} / R_{\mathrm{s}}^{3}$ and $\mu_{\mathrm{s}}$ are calculated from Eq. (34) for a fixed value $x=10$ as an example. Here we assumed $\dot{M}=10^{17} \mathrm{~g} \mathrm{~s}^{-1}$ and $R_{\mathrm{s}}=10 \mathrm{~km}$. We note that larger frequencies may occur at smaller distances, $x<10$.

model, based on a new preferred radius, the sonic radius. In this model, they assumed that near the neutron star there is a very narrow region of the disk in which the radial inflow velocity increases rapidly as the radius decreases. Such a sharp transition in the radial velocity of plasma flow from subsonic to supersonic happens at the "sonic point" radius, $r_{\text {sonic }}$. This radius tends to be close to the innermost stable circular orbit (ISCO) ${ }^{2}$, $r_{\text {ISCO }}$, however, radiative stresses may change its location, as required by the observation that the $\mathrm{kHz}$ QPO frequencies vary with time. Comparing the $\mathrm{HBO}$ and $\mathrm{kHz} \mathrm{QPO}$ frequencies, clearly $r_{\text {sonic }} \ll r_{\mathrm{A}}$, so part of the accreting matter must remain in near-Keplerian orbits well within $r_{\mathrm{A}}$. An important prediction of the sonic-point model is that $\Delta v=v_{2}-v_{1}$ is constant at the spin frequency of the neutron star $v_{\mathrm{s}}$, which is contrary to observations (Lamb \& Miller 2001). Accurate observations from the millisecond pulsar SAX J1808.4-3658 show that the frequency separation $\Delta v=195 \mathrm{~Hz}$, while the pulsar spin frequency is $v_{\mathrm{s}}=401 \mathrm{~Hz}$ (Wijnands et al. 2003; Chakrabarty et al. 2003). Nonetheless, further observations

${ }^{2}$ In general relativity, no stable orbital motion is possible within the innermost stable circular orbit (ISCO), $r_{\text {ISCO }}=$ $6 G M / c^{2} \approx 12.5 M_{1.4} M_{\odot} \mathrm{km}$. The frequency of orbital motion at the ISCO, the highest possible stable orbital frequency, is $v_{\mathrm{ISCO}} \approx$ $\left(1580 / M_{1.4} M_{\odot}\right) \mathrm{Hz}$. reveal that the frequency separation $\Delta v$ decreases when $v_{2}$ increases.

Stella \& Vietri $(1998,1999)$ proposed a relativistic precession model in which the high $\mathrm{kHz}$ QPO frequency $v_{2}$ is identified with the Keplerian frequency of an orbit in the disk (similar to the beat-frequency model) and the low $\mathrm{kHz}$ QPO frequency $v_{1}$ with the periastron precession of that orbit. Furthermore, in the relativistic model $\Delta v$ and $v_{\mathrm{s}}$ are not expected to be equal as in a beat-frequency interpretation. An interesting result of the relativistic precession model is that $\Delta v$ should decrease not only when $v_{2}$ increases (as observed) but also when it decreases sufficiently. However, in this model it is not clear how the X-ray flux is modulated at these frequencies.

Recently Zhang (2004) proposed a model for $\mathrm{kHz}$ QPOs based on magnetohydrodynamic (MHD) Alfvén oscillations in the disk. He introduced a new preferred radius, the quasi-sonic point radius, where the Alfvén velocity matches the orbital Keplerian velocity. The author suggested that the upper and lower $\mathrm{kHz}$ frequencies are the MHD Alfvén wave frequencies at the quasi-sonic point radius with low and high accreted material mass densities, respectively. In this model, the frequency separation will decrease when $v_{1}$ increases and/or sufficiently decreases. Although his results show good agreement with observations, several issues are left unresolved. For example, 
no mechanism is suggested to explain the excitation of Alfvén wave oscillations within the star's magnetosphere. More importantly, it is not clear how these Alfvén wave frequencies modulate the X-ray flux coming from the surface of the star.

In this paper, we propose a new model to explain QPOs in LMXBs based on interaction of accreting plasma with the neutron star magnetosphere. This interaction is a common feature in all accreting binary systems. Matter transfers from the companion star to the compact object. This matter is accelerated by the gravitational pull of the compact object and hits the magnetosphere of the star with a sonic/supersonic speed. The MHD interaction of the infalling plasma with the neutron star magnetosphere will alter not only the plasma flow toward the surface of the star, as assumed by current QPO models, but also the structure of the star's magnetosphere. The magnetic field of the neutron star is distorted inward by the infalling plasma of the Keplerian accretion flow. Since the gravity of the star confines the inward flow to a small solid angle $\sim 10^{-2}$ ster, the magnetic field of the star will be more compressed in the disk plane than in other areas (see Fig. 1). Furthermore, in a more realistic picture, one would expect that the highly accelerated plasma due to the infall process would be able to penetrate the magnetic field lines. Such material will be trapped by the magnetic field lines and produce enhanced density gradient regions within the magnetosphere ${ }^{3}$. However, due to the material's initial velocity, the penetrating material moves along the field lines, finally hitting the star's surface at the magnetic poles and producing the observed X-ray fluxes (Fig. 1). See Ghosh et al. (1977) for more details.

Besides modifying the geometry of the magnetosphere, the compressing action of the accretion flow can excite some plasma perturbations in the region of enhanced density gradients. This can be understood as the inward motion of the accretion flow being halted by the outward magnetic pressure at a certain distance from the star, the Alfvén radius (Ghosh et al. 1977). The accretion flow pushes the stellar magnetic field toward the star until the pressure of the infalling plasma $\rho v_{\mathrm{r}}^{2} / 2$ balances the magnetic pressure $B_{\mathrm{p}}^{2} / 8 \pi$. Here $\rho$ and $v_{\mathrm{r}}$ are the density and radial velocity of the infalling matter and $B_{\mathrm{p}}$ is the poloidal magnetic field at the disk plane. Therefore, any instability in the disk at the Alfvén radius would disturb such quasi-equilibrium configurations as well as the structure of the magnetosphere, possibly through the Rayleigh-Taylor and/or ballooning instability. As an example, the interaction of the solar wind with the Earth's magnetosphere excites resonant shear Alfvén waves, or field line resonances (FLRs), along the

\footnotetext{
${ }^{3}$ Note that the enhanced density gradient region is defined as follows: when we move outward from the surface of the star, the plasma density in the magnetosphere is more or less is constant, say $\rho_{0}$. At the point where magnetosphere interacts with the accretion disk (more likely at the disk's inner edge), however, there is a sudden increase in the magnetospheric plasma density, say $\rho>\rho_{0}$, due to the motion of accretion material along field lines. After this thin region (compared to the entire magnetosphere), the magnetospheric plasma density drops to $\rho_{0}$ again. Therefore, the enhanced density gradient region is not a region with a gradient in the plasma density only. It is a region with a different (higher) plasma density compared into the rest of the magnetosphere.
}

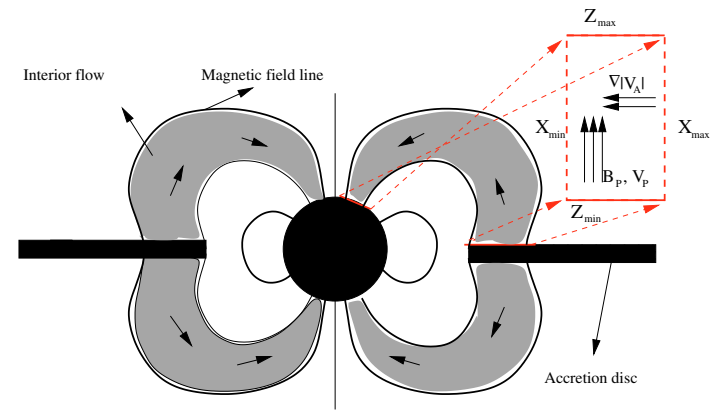

Fig. 1. A schematic and idealized side view of an accreting neutron star-disk system. The neutron star's strong gravity causes a very high velocity flow toward the magnetosphere. As a result, the magnetosphere is pushed inward in the disk plane but balloons outward in a direction away from the disk plane. Some of the plasma may leave the disk and flow along the field lines. The magnetic star-disk connection by a dipolar magnetosphere as well as the polar accretion flow along the field lines are part of a reasonable but simplified picture of magnetized star-disk systems in general. The dashed box represents the model that we considered in this paper. $z_{\max }>0$ represents the surface of the star, $z_{\min }>0$ is on the disk plane, $x_{\min }$ and $x_{\max }$ are embedded in the density gradient region. The directions of the ambient magnetic field and the plasma flows in the box are indicated by the vertical arrows, while the direction of the gradient in the Alfvén speed is shown by the horizontal arrows.

magnetic field lines (Samson 1991). As a result, such Alfvén waves may be excited by the compressive accreting plasma in the magnetosphere of an accreting neutron star with the accretion flow playing the role of the solar wind.

The plan of this paper is as follows. We outline the occurrence of resonant coupling in a magnetized plasma in Sect. 2. We review the excitation of shear Alfvén waves by studying linear perturbations in MHD. Next, the occurrence of FLRs, as a result of a resonant coupling between the compressional and shear Alfvén waves, is discussed. To illustrate the basic features of FLRs, the excitation of these resonances in a rectilinear magnetic field is considered. In Sect. 3 , in order to consider a more realistic model for an accreting neutron star, we study the influence of the ambient flow along the field line on the excitation of FLRs. We demonstrate that in this case the eigenfrequency of the Alfvén modes is modulated by the velocity of the field-aligned plasma flow. Depending on the field line where the resonance occurs, the eigenfrequency of the FLR is in the range of several hundred $\mathrm{Hz}$ to $\mathrm{kHz}$. Furthermore, in the presence of this flow, the plasma displacement parallel to the magnetic field lines is non-zero. This is very important because these displacements, which are missing in the case of zero ambient flow, might be responsible for modulating the motion of infalling material toward the magnetic poles and producing the observed X-ray fluxes. Existence of this non-zero parallel component distinguishes our study from other related investigations in which the modulation of X-ray flux in observed QPOs is fairly unclear or ambiguous. A possible occurrence of more than one peak in the power spectrum is also discussed. In addition, we show that the observed 2:3 frequency ratio of QPOs is a natural result of our model. Section 4 is devoted to summarizing our results and further discussion. 


\section{The magnetospheric model}

\subsection{Magneto-hydrodynamic waves}

In general, the dynamics of an ideal magnetized plasma is described by plasma density $\rho$, plasma pressure $p$, gravitational potential $\Phi$, velocity vector $\boldsymbol{v}$ and magnetic field $\boldsymbol{B}$ :

$$
\begin{aligned}
& \partial \boldsymbol{v} / \partial t+\boldsymbol{v} \cdot \boldsymbol{\nabla} \boldsymbol{v}=-\boldsymbol{\nabla} p / \rho-\frac{1}{4 \pi \rho} \boldsymbol{B} \times(\boldsymbol{\nabla} \times \boldsymbol{B})-\boldsymbol{\nabla} \Phi, \\
& \partial \rho / \partial t+\boldsymbol{\nabla} \cdot(\rho \boldsymbol{v})=0, \\
& \partial \boldsymbol{B} / \partial t=\boldsymbol{\nabla} \times(\boldsymbol{v} \times \boldsymbol{B}), \\
& \boldsymbol{\nabla} \cdot \boldsymbol{B}=0 .
\end{aligned}
$$

An adiabatic equation of state with an adiabatic index $\gamma$, $p / \rho^{\gamma}=$ const., is assumed in this paper in order to complete the above equations.

In a steady state (i.e. $\partial / \partial t=0$ ), Eqs. (1) have been studied in detail in connection with the problem of stellar winds from rotating magnetic stars (Mestel 1968a,b) and in connection with diffusing/flowing plasma into magnetospheres in accreting neutron stars (Elsner \& Lamb 1977, 1984; Ghosh et al. 1977). Obviously, in the case of accreting neutron stars, $v$ represents the inflow velocity of matter accreted to stars, while in stars with a stellar wind it represents the plasma outflow velocity.

Consider an axisymmetric system consisting of a rotating star with a constant angular velocity $\Omega_{\mathrm{s}}$ along the $z$-axis and a magnetic field whose symmetry axis is aligned with the rotation axis of the star. By decomposing the velocity and magnetic field vectors into poloidal and toroidal components:

$\boldsymbol{v}=\boldsymbol{v}_{\mathrm{p}}+\Omega \varpi \hat{\boldsymbol{\phi}}, \quad \boldsymbol{B}=\boldsymbol{B}_{\mathrm{p}}+B_{\phi} \hat{\boldsymbol{\phi}}$,

one can obtain

$\boldsymbol{v}_{\mathrm{p}}=(f / \rho) \boldsymbol{B}_{\mathrm{p}}$,

$\Omega=\Omega_{\mathrm{s}}+(f / \rho)\left(B_{\phi} / \varpi\right)$

where $f$ is the mass flux along a magnetic flux tube of unit flux (Ghosh et al. 1977). The subscripts $p$ and $\phi$ denote poloidal and toroidal components, respectively, $\Omega$ is the angular velocity of the plasma, $\varpi$ is the distance from the axis of the rotation, and $\hat{\boldsymbol{\phi}}$ is a unit toroidal vector. We note that both $f$ and $\Omega_{\mathrm{s}}$ are constant along a given field line. Equation (4) can be rewritten as

$\Omega=\Omega_{\mathrm{s}}+\left(v_{\mathrm{p}} / \varpi\right)\left(B_{\phi} / B_{\mathrm{p}}\right)$,

where $v_{\mathrm{p}}=\boldsymbol{v}_{\mathrm{p}} \cdot \hat{\boldsymbol{p}}$ is the magnitude of velocity along the poloidal magnetic field $\boldsymbol{B}_{\mathrm{p}}$ with magnitude $B_{\mathrm{p}}$ and $\hat{\boldsymbol{p}}=\boldsymbol{B}_{\mathrm{p}} / B_{\mathrm{p}}$. Furthermore, Ghosh et al. (1977) have shown that at distances close to the Alfvén radius, the magnitude of the poloidal component of the inflow velocity (for accreting systems) $v_{\mathrm{p}}$ approaches the poloidal Alfvén speed $v_{\mathrm{A}}$, i.e. ${ }^{4}$

$v_{\mathrm{p}}^{2}\left(r_{\mathrm{A}}\right)=v_{\mathrm{A}}^{2}\left(r_{\mathrm{A}}\right)$

${ }^{4}$ In order to estimate the poloidal component of the inflow velocity $v_{\mathrm{p}}$, one needs to integrate the momentum Eq. (1a):

$(1 / 2)\left(v_{\mathrm{p}}^{2}+\Omega^{2} \varpi^{2}\right)-\Omega_{\mathrm{s}} \Omega \varpi^{2}-G M / r=$ constant along a given field line,
The possible perturbations of a magnetized plasma and MHD waves are found by specifying the equilibrium configuration of the star and then solving the perturbed Eqs. (1). This is a nontrivial problem that has been addressed by several investigators. However, in this paper we will be interested in the propagation of shear Alfvén waves in the star's magnetosphere and the resulting FLRs.

\subsection{Field line resonances}

In MHD, waves can propagate in three different modes including shear Alfvén waves, and the fast and slow compressional modes (Landau \& Lifshitz 1992). In a homogeneous plasma, one can easily show that these three modes are linearly independent. However, in an inhomogeneous medium these three modes can be coupled, yielding either a resonant coupling (Southwood 1974; Hasegawa 1976), or an unstable ballooning mode (Ohtani \& Tamao 1993; Liu 1997). FLRs result from the coupling of the fast compressional and the shear Alfvén modes ${ }^{5}$ whereas the ballooning instability results from the coupling of the slow compressional and the shear Alfvén modes. However, in the cold-plasma approximation (which is appropriate for neutron star magnetospheres), the slow compressional wave does not exist. FLRs are standing waves that are stimulated in a pulsar with gradients in the Alfvén speed transverse to the ambient B-field, particularly within density boundary layers (a parallel gradient in the Alfvén speed is also included). Efficient coupling between the shear Alfvén wave and the fast compressional wave can produce a relatively narrow FLR spectrum, even when the driver is broadband.

The linear theory of FLRs was developed by Chen \& Hasegawa (1974) and Southwood (1974) and applied to auroral phenomena by Hasegawa (1976). Samson et al. (2003) developed a nonlinear model with a nonlocal electron conductivity to explain the evolution of field-aligned potential drops and electron acceleration to form auroral arcs. They studied the possible coupling between the fast compressional mode and the shear Alfvén mode in an inhomogeneous plasma with a radial gradient in the Alfvén velocity $v_{\mathrm{A}}=B / \sqrt{4 \pi \rho}$. For further discussion of mode conversion for shear Alfvén waves see for instance Stix (1992), and for an example of computational models see Rickard \& Wright (1994). Analytic solutions for toroidal FLRs in dipole magnetic fields can be found in Taylor \& Walker (1984).

where $M$ is the mass of the neutron star (Mestel 1968a,b; Ghosh et al. 1977). In the above equation the pressure term is neglected. Equation (6) shows conservation of energy in a frame corotating with the star, while in a nonrotating frame the extra term $\Omega_{\mathrm{s}} \Omega \varpi^{2}$ appears, that represents the work done by the magnetic field on the flowing plasma. However, as argued by Ghosh et al. (1977) the poloidal velocity in the inner magnetosphere where $r \ll r_{\mathrm{A}}$ is nearly equal to the free-fall velocity, i.e.

$v_{\mathrm{p}} \sim(2 G M / r)^{1 / 2}$.

5 In the cold-plasma approximation, the fast compressional wave (fast magnetosonic wave) is also called the compressional Alfvén wave. 
To illustrate the resonant coupling between the fast compressional mode and the shear Alfvén mode we study the excitation of FLRs in an inhomogeneous plasma in a rectilinear magnetic field model.

\subsubsection{FLRs in a rectilinear magnetic field}

Plasma dynamics can often be described by the adiabatic ideal MHD equations:

$\rho(\partial / \partial t+\boldsymbol{v} \cdot \boldsymbol{\nabla}) \boldsymbol{v}=-\boldsymbol{\nabla} p-\frac{1}{4 \pi} \boldsymbol{B} \times(\boldsymbol{\nabla} \times \boldsymbol{B})$,

$\partial \boldsymbol{B} / \partial t=\boldsymbol{\nabla} \times(\boldsymbol{v} \times \boldsymbol{B})$,

$\boldsymbol{\nabla} \cdot \boldsymbol{B}=0$

$\partial \rho / \partial t+\boldsymbol{\nabla} \cdot(\rho \boldsymbol{v})=0$,

$\mathrm{d}\left(p \rho^{-\gamma}\right) / \mathrm{d} t=0$,

where we neglect the effect of gravitational attraction on the plasma (see Eqs. (1), for comparison). Introducing Eulerian perturbations to the ambient quantities in the form

$p=p_{0}+\delta p, \rho=\rho_{0}+\delta \rho$,

$\boldsymbol{B}=\boldsymbol{B}_{0}+\delta \boldsymbol{B}, \boldsymbol{v}=\delta \boldsymbol{v}=\partial \boldsymbol{\xi} / \partial t$,

we can derive a linear wave equation by simplifying Eqs. (9) up to the first order in the perturbations. We choose coordinates such that the ambient magnetic field is in the $z$-direction. Without loss of generality, we assume that the gradients in all the unperturbed quantities are in the $x$-direction. Setting the plasma displacement as

$\boldsymbol{\xi}(\boldsymbol{r}, t)=\boldsymbol{\xi}(x) \mathrm{e}^{-\mathrm{i}\left(\omega t-k_{y} y-k_{z} z\right)}$,

$\delta \boldsymbol{B}(\boldsymbol{r}, t)=\delta \boldsymbol{B}(x) \mathrm{e}^{-\mathrm{i}\left(\omega t-k_{y} y-k_{z}\right)}$,

$\delta \rho(\boldsymbol{r}, t)=\delta \rho(x) \mathrm{e}^{-\mathrm{i}\left(\omega t-k_{y} y-k_{z} z\right)}$,

$\delta p(\boldsymbol{r}, t)=\delta p(x) \mathrm{e}^{-\mathrm{i}\left(\omega t-k_{y} y-k_{z} z\right)}$,

Eqs. (9) reduce to (Harrold \& Samson 1992)

$\frac{\mathrm{d}^{2} \xi_{x}}{\mathrm{~d} x^{2}}+\frac{F^{\prime}(x)}{F(x)} \frac{\mathrm{d} \xi_{x}}{\mathrm{~d} x}+G(x) \xi_{x}=0$

with

$G(x)=\frac{\omega^{2}\left(\omega^{2}-\left(v_{\mathrm{S}}^{2}+v_{\mathrm{A}}^{2}\right)\left(k_{y}^{2}+k_{z}^{2}\right)\right)+k_{z}^{2}\left(k_{y}^{2}+k_{z}^{2}\right) v_{\mathrm{A}}^{2} v_{\mathrm{S}}^{2}}{\left(v_{\mathrm{S}}^{2}+v_{\mathrm{A}}^{2}\right) \omega^{2}-k_{z}^{2} v_{\mathrm{A}}^{2} v_{\mathrm{S}}^{2}}$,

and

$F(x)=\frac{\rho_{0}\left(\omega^{2}-k_{z}^{2} v_{\mathrm{A}}^{2}\right)}{G(x)}$,

where $v_{\mathrm{A}}^{2}(x)=B_{0}^{2} /\left(4 \pi \rho_{0}\right)$ and $v_{\mathrm{S}}^{2}(x)=\gamma p_{0} / \rho_{0}$, and ${ }^{\prime}=\mathrm{d} / \mathrm{d} x$. Here we assumed that the total pressure (fluid+field) $P_{0}=p_{0}+$ $B_{0}^{2} /(8 \pi)$ is constant. Equation (11) yields two turning points at $G(x)=0$, and two resonances at $F(x)=0$. Close to the resonance positions, Eq. (11) can be approximated by

$\frac{\mathrm{d}^{2} \xi_{x}}{\mathrm{~d} x^{2}}+\frac{1}{x-x_{0}} \frac{\mathrm{d} \xi_{x}}{\mathrm{~d} x}+G(x) \xi_{x}=0$,

where $x_{0}$ is the position where the resonance occurs.

In the case of a strong magnetic field, or for a cold plasma (i.e. $p_{\text {fluid }} / p_{\text {magnetic }} \ll 1$ ), one can put $v_{\mathrm{S}} \simeq 0$. Then $G(x)$ and $F(x)$, Eqs. (12) and (13), reduce to

$G(x)=\frac{\omega^{2}}{v_{\mathrm{A}}^{2}}-k_{y}^{2}-k_{z}^{2}$

and

$F(x)=\frac{v_{\AA}^{2} \rho_{0}\left(\omega^{2}-k_{z}^{2} v_{\AA}^{2}\right)}{\omega^{2}-\left(k_{y}^{2}+k_{z}^{2}\right) v_{\mathrm{A}}^{2}}$.

In this case Eq. (11) has only one resonance (the Alfvén resonance) at

$\omega^{2}-k_{z}^{2} v_{\mathrm{A}}^{2}=0$,

which corresponds to the dispersion relation for the shear Alfvén wave along the field line. In the above calculations, four boundary conditions are assumed. In the $\mathrm{x}$-direction the boundaries, say at $x_{\min }=0$ and $x_{\max }=x_{\mathrm{m}}$ are considered perfectly reflecting (i.e. $\xi_{x}=0$ at boundaries). Further, we assume that neutron star and accretion disk surfaces are perfectly conducting so that the displacement $\xi$ vanishes at these surfaces. In the rectilinear model we represented these boundaries by planes at constant $z$, i.e. $z_{\min }>0$ on the disk and $z_{\max }>0$ on the star's surface (see Fig. 1).

Briefly, the FLR mechanism can be outlined as follows: the incoming compressional wave hits the stellar magnetosphere and reaches a field line (or a magnetospheric shell) at which the frequency of the incoming wave matches the eigenfrequency of the standing shear Alfvén wave along that particular filed line. The resulting forced-oscillating system causes the amplitude of the shear Alfvén wave to grow in time, forming FLRs.

The FLR mechanism is generic and likely to occur in many astrophysical magnetospheres. As a result, one would expect that FLRs likely occur not only in the Earth's magnetosphere but also in the magnetospheres of accreting neutron stars. In the case of the Earth's magnetosphere, the source of energy for the FLRs is the interaction of the solar wind with the magnetosphere (Harrold \& Samson 1992). In accreting neutron stars, the accreted plasma interacts with the stars' magnetosphere, allowing the compressional mode to propagate into the magnetosphere and flow along the field lines toward the magnetic poles. Such a compressional action of the accretion flow can excite resonant shear Alfvén waves in the enhanced density regions filled by plasma flowing along the field lines. In Sect. 3 we consider this mechanism more carefully to address its possible relation to the QPOs observed in accreting neutron stars in LMXBs. 


\section{FLRs in the presence of ambient flow (along the magnetospheric field lines)}

As discussed above, FLRs have been used to model electron acceleration and auroral arcs in the Earth's magnetosphere. Although FLRs are likely to be excited in any magnetospheric system with an input of compressional energy, one must carefully evaluate the differences between the Earth's and neutron star magnetospheres. In neutron star magnetospheres, for example, the strong magnetic field of the neutron star, the rapid stellar rotation and the intense radiation pressure from the stellar surface should be considered.

In the case of the Earth's magnetosphere, due to the small gravitational attraction of the Earth and also its large distance from the Sun, the solar wind impacts the whole Sunward side of the geomagnetosphere. This supersonic solar wind produces the so-called bow shock structure at the outer boundary of the geomagnetosphere. On the contrary, the strong gravity of the neutron star creates a converging flow that is supersonic long before the flow hits the star's magnetosphere. Such a localized flow is able to change the structure of the magnetosphere in local areas, particularly in the equatorial plane. In addition, the highly variable nature of the exterior flow can change the magnetosphere's structure dramatically in time. The large flux of plasma stresses the star's outer magnetosphere and creates a relatively high plasma density in this region. The plasma then flows along the field lines, an interior flow, until it hits the star's surface near the magnetic poles (see Fig. 1). In this section we study the excitation of FLRs by considering such plasma flows in the neutron star magnetosphere. In the context of the Earth's magnetosphere, however, Harrold \& Samson (1992) studied the resonant excitation of Alfvén waves by surface waves excited by the solar wind and plasma flows in the Earth's magnetosheath.

The presence of a flow $\boldsymbol{v}$ in the plasma adds more modes to the plasma waves. In general, such flows are a combination of plasma flow along the magnetic field lines, $\boldsymbol{v}_{\mathrm{p}}$, and rotational motion of the plasma around the star, with angular velocity $\mathbf{\Omega}$, i.e. $\boldsymbol{v}=\boldsymbol{v}_{\mathrm{p}}+\boldsymbol{\Omega} \times \boldsymbol{r}$ (see Eq. (2)). As we shall see in Eq. (22a), the existence of $\boldsymbol{v}_{\mathrm{p}}$ alone induces a non-zero parallel (relative to the direction of the magnetic field) component of displacement that vanishes in a cold plasma if $\boldsymbol{v}_{\mathrm{p}}=0$. We note that this component can be responsible for modulating the infalling plasma flow near the star's surface with FLRs' frequencies and producing the observed X-ray fluxes, see Eq. (22a) below. We will return to this point later.

In a strong magnetic field, the usual definition of the Alfvén velocity, $v_{\mathrm{A}}^{2}=B^{2} /(4 \pi \rho)$, is not correct as with this nonrelativistic definition, velocities can be larger than the speed of light, $c$. The correct definition, the relativistic Alfvén velocity, uses energy density $\epsilon$ relative to $c^{2}$, which for an ideal gas reads

$h \equiv \frac{\epsilon}{c^{2}}=\frac{c^{2} \rho+\gamma p /(\gamma-1)+B^{2} /(4 \pi)}{c^{2}}$

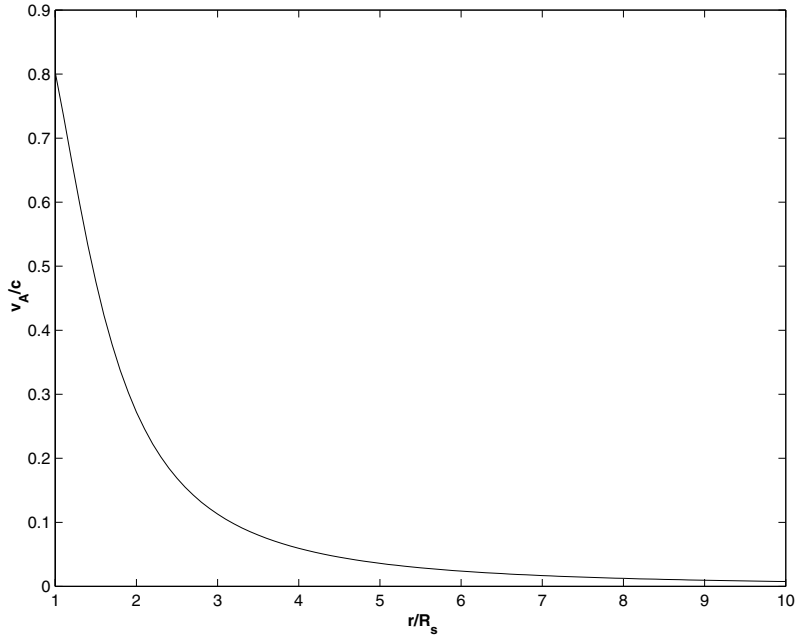

Fig. 2. Radial profile of the relativistic Alfvén speed $v_{\mathrm{A}}$ (in units of speed of light) in the equatorial plane, see Eq. (30). $R_{\mathrm{S}}$ is the radius of the neutron star. Here we assumed $p=0, R_{\mathrm{s}}=10 \mathrm{~km}, M=M_{\odot}$, $\dot{M}=10^{17} \mathrm{~g} \mathrm{~s}^{-1}$ and $\mu=10^{26} \mathrm{G} \mathrm{cm}^{3}$.

rather than the plasma density $\rho$. Consequently the (relativistic) Alfvén velocity is

$v_{\mathrm{A}}^{2}=B^{2} /(4 \pi h)=\frac{c^{2}}{c^{2} \rho+\gamma p /(\gamma-1)+B^{2} /(4 \pi)} \frac{B^{2}}{4 \pi}$.

In Fig. 2, we plot radial profiles of the relativistic Alfvén velocity $v_{\mathrm{A}}$ in the equatorial plane (assuming $p=0$ ). As shown in this figure, close to the star the relativistic Alfvén velocity approaches the speed of light, i.e. $v_{\mathrm{A}} \sim c$. This definition for the Alfvén velocity is used extensively in studies of relativistic jet dynamics in black hole systems and quasars (see for an example Mizuno et al. 2004). Again, we use a linear approximation to obtain a dispersion relation with resonant coupling.

The linearized perturbed magnetohydrodynamic equations in the presence of an ambient flow can be obtained from Eqs. (9) as

$$
\begin{aligned}
& h\left(\frac{\partial \delta \boldsymbol{v}}{\partial t}+\boldsymbol{v} \cdot \boldsymbol{\nabla} \delta \boldsymbol{v}+\delta \boldsymbol{v} \cdot \boldsymbol{\nabla v}\right)=-\boldsymbol{\nabla} \delta P+\frac{\boldsymbol{\nabla} P}{h} \delta h \\
& +\frac{1}{4 \pi}(\delta \boldsymbol{B} \cdot \boldsymbol{\nabla} \boldsymbol{B}+\boldsymbol{B} \cdot \boldsymbol{\nabla} \delta \boldsymbol{B}), \\
& \frac{\partial \delta \boldsymbol{B}}{\partial t}=\boldsymbol{\nabla} \times(\delta \boldsymbol{v} \times \boldsymbol{B}+\boldsymbol{v} \times \delta \boldsymbol{B}), \\
& \boldsymbol{\nabla} \cdot \delta \boldsymbol{B}=0, \\
& \left(\frac{\partial}{\partial t}+\boldsymbol{v} \cdot \boldsymbol{\nabla}\right) \delta p+\delta \boldsymbol{v} \cdot \boldsymbol{\nabla} p=-\gamma(\delta p \boldsymbol{\nabla} \cdot \boldsymbol{v}+p \boldsymbol{\nabla} \cdot \delta \boldsymbol{v}),
\end{aligned}
$$

where $\delta \boldsymbol{v}=\partial \boldsymbol{\xi} / \partial t$. $P=p+B^{2} /(8 \pi)$ and $\delta P=\delta p+\boldsymbol{B} \cdot \delta \boldsymbol{B} /(4 \pi)$ are the total unperturbed and perturbed plasma pressure (fluid + field), respectively. Here $h, p, v$, and $\boldsymbol{B}$ are the unperturbed quantities while $\delta h, \delta p, \delta \boldsymbol{v}$, and $\delta \boldsymbol{B}$ are the perturbed quantities. Furthermore, we consider the slow rotation approximation and neglect both the toroidal field $B_{\phi}$ and velocity $v_{\phi}$, Eq. (2), for simplicity. Such assumptions may not be entirely accurate, but simplify our calculations significantly.

To treat the problem analytically, we again consider a rectilinear magnetic field configuration. Although this configuration may not be suitable for an accreting neutron star, it may 
describe QPOs. Separating Eqs. (20) into parallel and perpendicular components relative to the ambient magnetic field and assuming perturbed quantities in the form of

$\delta(\boldsymbol{r}, t)=\delta\left(\boldsymbol{r}_{\perp}\right) \mathrm{e}^{-\mathrm{i}\left(\omega t-k_{\|} r_{\mu}\right)}$,

we find

$$
\begin{aligned}
& -\mathrm{i} \omega_{\mathrm{D}} \xi_{\|}=-\frac{k_{\|} v_{\mathrm{s}}^{2}}{\omega_{\mathrm{D}}} \nabla \cdot \boldsymbol{\xi}-\left(\boldsymbol{\xi}_{\perp} \cdot \boldsymbol{\nabla}_{\perp}\right) v_{\|}, \\
& \left(\omega_{\mathrm{D}}^{2}-k_{\|}^{2} v_{\mathrm{A}}^{2}\right) \boldsymbol{\xi}_{\perp}=\frac{\omega_{\mathrm{D}}}{\omega} \frac{\nabla_{\perp} \delta P}{h}-\frac{\omega_{\mathrm{D}}}{\omega} \frac{\nabla_{\perp} P}{h^{2}} \delta h, \\
& -\mathrm{i} \omega_{\mathrm{D}} \delta B_{\|}=\mathrm{i} \omega B\left(\boldsymbol{\nabla}_{\perp} \cdot \boldsymbol{\xi}_{\perp}\right)+\mathrm{i} \omega\left(\boldsymbol{\xi}_{\perp} \cdot \boldsymbol{\nabla}_{\perp}\right) B \\
& \quad+\left(\delta \boldsymbol{B}_{\perp} \cdot \boldsymbol{\nabla}_{\perp}\right) v_{\|}, \quad(\mathrm{using} \boldsymbol{\nabla} \cdot \delta \boldsymbol{B}=0) \\
& -\mathrm{i} \omega_{\mathrm{D}} \delta \boldsymbol{B}_{\perp}=\omega k_{\|} B \boldsymbol{\xi}_{\perp}, \quad(\operatorname{using} \boldsymbol{\nabla} \cdot \boldsymbol{B}=0) \\
& -\mathrm{i} \omega_{\mathrm{D}} \delta p=\mathrm{i} \omega\left(\boldsymbol{\xi}_{\perp} \cdot \nabla_{\perp}\right) p-\gamma \omega p\left(k_{\|} \xi_{\|}-\mathrm{i} \nabla_{\perp} \cdot \boldsymbol{\xi}_{\perp}\right), \\
& -\frac{\delta P}{h}=\frac{\omega}{\omega_{\mathrm{D}}}\left(v_{\mathrm{s}}^{2}+v_{\mathrm{A}}^{2}\right) \boldsymbol{\nabla} \cdot \boldsymbol{\xi}-\frac{\mathrm{i} \omega k_{\|} v_{\mathrm{A}}^{2}}{\omega_{\mathrm{D}}} \xi_{\|} \\
& \quad+\frac{\omega k_{\|} v_{\mathrm{A}}^{2}}{\omega_{\mathrm{D}}^{2}}\left(\boldsymbol{\xi}_{\perp} \cdot \boldsymbol{\nabla}_{\perp}\right) v_{\|},
\end{aligned}
$$

where $\omega_{\mathrm{D}}=\omega-k_{\|} v_{\|}$is the Doppler shifted frequency, $\omega$ is the eigenfrequency, $k_{\|}$is the wave number in the parallel direction, $\boldsymbol{v}_{\mathrm{p}}=v_{\|} \boldsymbol{B} / B, v_{\mathrm{s}}=\sqrt{\gamma p / h}$ is the sound velocity ${ }^{6}$. Note that we assumed that all ambient quantities are functions of $\boldsymbol{r}_{\perp}$ only. In order to derive Eq. (22), Eq. (22a) uses Eqs. (22d) and (22e), Eq. (22b) uses Eq. (22d), and Eq. (22f) is a combination of Eqs. (22c), (22d), and (22e). Equation (22a) shows that the plasma displacement parallel to the ambient field does not vanish even in the cold plasma approximation $(p=0)^{7}$. The non-zero $\xi_{\|}$can affect and then modulate the motion of plasma along the field lines. This modulation will occur at the frequency of the resonant shear Alfvén waves $\omega$ and should be observed in the X-ray fluxes.

We set the magnetic field in the $z$-direction with gradients in the ambient parameters in the $x$-direction $\left(\boldsymbol{r}_{\perp}\right)$ only, i.e. $v_{\|}\left(\boldsymbol{r}_{\perp}\right)=v_{\mathrm{p}}(x)=v_{z}(x)$, and further

$\delta(\boldsymbol{r}, t)=\delta(x) \mathrm{e}^{-\mathrm{i}\left(\omega t-k_{y} y-k_{z} z\right)}$.

Here we assumed that the perturbed quantities have sinusoidal dependencies in both $y$ - and $z$-directions. Equations (22a), $(22 \mathrm{~b})$ and $(22 \mathrm{f})$ in the cold plasma approximation $(p=0)$ can then be combined into one differential equation

$\frac{\mathrm{d}^{2} \xi_{x}}{\mathrm{~d} x^{2}}+\frac{\omega_{\mathrm{D}} \Xi^{\prime}(x)+k_{z} v_{\mathrm{p}}^{\prime} \Xi(x)}{\omega_{\mathrm{D}} \Xi(x)} \frac{\mathrm{d} \xi_{x}}{\mathrm{~d} x}$

$+\left(\frac{k_{z} v_{\mathrm{p}}^{\prime} \Xi^{\prime}(x)}{\omega_{\mathrm{D}} \Xi(x)}+\left(\frac{k_{z} v_{\mathrm{p}}^{\prime}}{\omega_{\mathrm{D}}}\right)^{\prime}+\frac{\omega_{\mathrm{D}}^{2}-\left(k_{y}^{2}+k_{z}^{2}\right) v_{\mathrm{A}}^{2}}{v_{\mathrm{A}}^{2}}\right) \xi_{x}=0$,

where $\omega_{\mathrm{D}}=\omega-k_{z} v_{\mathrm{p}}$ and

$F(x) \equiv \omega_{\mathrm{D}} \Xi(x)=\frac{\omega_{\mathrm{D}}^{2}-k_{z}^{2} v_{\mathrm{A}}^{2}}{\omega_{\mathrm{D}}^{2}-\left(k_{y}^{2}+k_{z}^{2}\right) v_{\mathrm{A}}^{2}} h v_{\mathrm{A}}^{2}$.

\footnotetext{
${ }^{6}$ To obtain Eq. (22f) we assumed that at equilibrium $\boldsymbol{\nabla} P=\boldsymbol{\nabla}(p+$ $\left.B^{2} /(8 \pi)\right)=0$. This is valid for a non-rotating or very slowly rotating star.

${ }^{7}$ In the case of zero ambient flow, $v_{\|}=0$, the parallel displacement $\xi_{\|}$vanishes in the cold plasma.
}

Note that here $v_{\mathrm{p}}=v_{\mathrm{p}}(x)$ and $v_{\mathrm{A}}=v_{\mathrm{A}}(x)$ are functions of $x$. For $v_{\mathrm{p}}=0$ Eqs. (24) and (25) reduce to the one obtained in Sect. 2.2.1, Eqs. (11), (15) and (16) with $\rho_{0}$ replaced by $h$, as expected.

Mathematically our result is similar to the one obtained by Harrold \& Samson (1992), however, they considered a different model in which flow in the Earth's magnetosheath is perpendicular to the B-field and there is no ambient flow in the region of FLRs. For a constant flow along magnetic field lines, i.e. $v_{\mathrm{p}}^{\prime}(x)=0$, Eq. (24) reduces to

$\frac{\mathrm{d}^{2} \xi_{x}}{\mathrm{~d} x^{2}}+\frac{F^{\prime}(x)}{F(x)} \frac{\mathrm{d} \xi_{x}}{\mathrm{~d} x}+\frac{\omega_{\mathrm{D}}^{2}-\left(k_{y}^{2}+k_{z}^{2}\right) v_{\mathrm{A}}^{2}}{v_{\mathrm{A}}^{2}} \xi_{x}=0$.

Equation (26) has a singular point at $F=0$. The condition $F=0$ yields the following modes:

$\omega^{ \pm}=k_{z}\left(v_{\mathrm{p}} \pm v_{\mathrm{A}}\right)$.

The resulting modes are a combination of a compressional mode $k_{z} v_{\mathrm{p}}$ due to the plasma flow and a shear Alfvén mode $k_{z} v_{\mathrm{A}}$. They produce two frequency peaks in the spectrum that vary with time if the velocities change with time. The value of $k_{z} \sim \pi / L$ where $L$ is the length of the field line and is of the order of the radius of the neutron star, $L \sim R_{\mathrm{S}}$. Therefore, for $R_{\mathrm{S}} \sim 10^{6} \mathrm{~cm}$ and $v_{\mathrm{A}} \sim v_{\mathrm{p}} \sim 1.5 \times 10^{8} \mathrm{~cm} \mathrm{~s}^{-1}$ we get $\omega^{+} \sim 1000 \mathrm{~Hz}$, which is comparable to the frequencies of the observed QPOs. In the limit $v_{\mathrm{p}} \ll v_{\mathrm{A}}$ these modes become a single mode of a regular Alfvén resonance. In the case of $v_{\mathrm{A}} \ll c$ this mode becomes consistent with a single mode (17). For a superalfvénic flow, i.e. $v_{\mathrm{A}} \ll v_{\mathrm{p}}$, however, the MHD Alfvén waves and the resulting FLRs are suppressed due to propagation of the MHD wave, $k_{z} v_{\mathrm{p}}$. In the case of superalfvénic plasma flow, one has

$$
F(x) \simeq \frac{h k_{z}^{2} v_{\mathrm{A}}^{2}}{k_{y}^{2}+k_{z}^{2}}, \text { as } \omega \rightarrow k_{z} v_{\mathrm{p}},
$$

which shows that Eq. (26) is no longer singular. Therefore, no FLRs are likely to occur in this case.

As a result, we expect that the two FLR peaks (Eq. (27)) occur where $v_{\mathrm{p}}$ and $v_{\mathrm{A}}$ are more or less comparable (i.e. of the same order of magnitude). As we now show, using the flow velocity and the Alfvén velocity definitions below, one might expect that the FLRs likely occur at $R_{\mathrm{S}}<r<100 R_{\mathrm{s}}$.

Approximating the plasma inflow velocity by the free fall velocity $v_{\mathrm{p}} \sim v_{\mathrm{fff}}(r)$ and $v_{\mathrm{A}} \sim B(r) / \sqrt{4 \pi \rho_{\mathrm{ff}}}$ where $\rho_{\mathrm{ff}}=$ $\dot{M} /\left(v_{\mathrm{ff}} 4 \pi r^{2}\right)$ is the free fall mass density, one can rewrite Eq. (27) as:

$$
\begin{aligned}
\omega^{ \pm}(r) & \simeq k_{z}\left((2 G M / r)^{1 / 2} \pm B(r) / \sqrt{4 \pi \rho_{\mathrm{fff}}(r)}\right), \\
& \simeq k_{z}\left(v_{\mathrm{p}}\left(R_{\mathrm{s}}\right) x^{-1 / 2} \pm v_{\mathrm{A}}\left(R_{\mathrm{s}}\right) x^{-9 / 4}\right),
\end{aligned}
$$

where $v_{\mathrm{p}}\left(R_{\mathrm{s}}\right)=\left(2 G M / R_{\mathrm{s}}\right)^{1 / 2} \simeq 1.6 \times 10^{10}\left(M / M_{\odot}\right)^{1 / 2}$ $\left(R_{\mathrm{s}} / 10 \mathrm{~km}\right)^{-1 / 2} \mathrm{~cm} \mathrm{~s}^{-1}$ and $v_{\mathrm{A}}\left(R_{\mathrm{S}}\right)=B\left(R_{\mathrm{S}}\right) / \sqrt{4 \pi \rho_{\mathrm{ff}}\left(R_{\mathrm{S}}\right)} \simeq 4 \times$ $10^{10} \mu_{26} \dot{M}_{17}^{-1 / 2}\left(M / M_{\odot}\right)^{1 / 4}\left(R_{\mathrm{s}} / 10 \mathrm{~km}\right)^{-9 / 4} \mathrm{~cm} \mathrm{~s}^{-1}$ are inflow and 


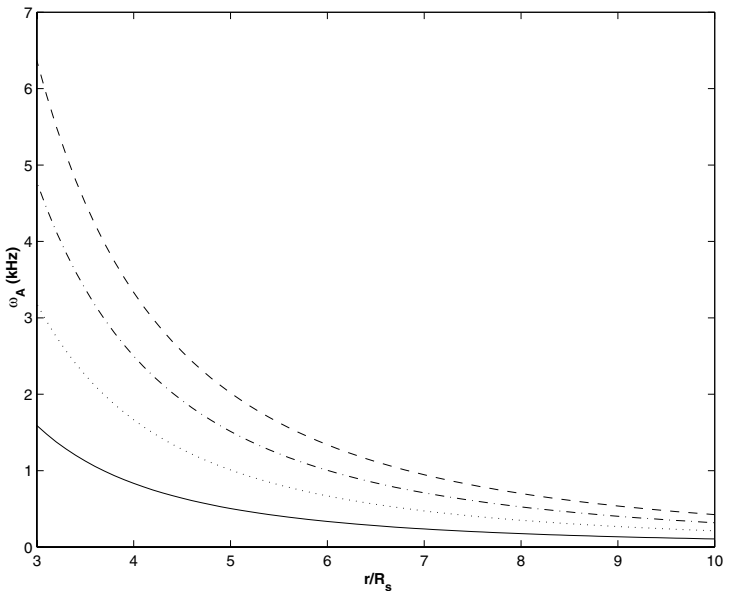

Fig. 3. Frequency of the regular Alfvén resonance $\left(v_{\mathrm{p}}=0\right)$ as function of $r, \omega_{\mathrm{A}}=k_{z} v_{\mathrm{A}}\left(R_{\mathrm{s}}\right)\left(r / R_{\mathrm{s}}\right)^{-9 / 4}$. The closer to the star the higher the frequency. From bottom to top $v_{\mathrm{A}}\left(R_{\mathrm{S}}\right)=.2 c, .4 c, .6 c$ and $.8 c$, respectively. Here $R_{\mathrm{S}}=10 \mathrm{~km}$, and $k_{z}=\pi / R_{\mathrm{s}}$.

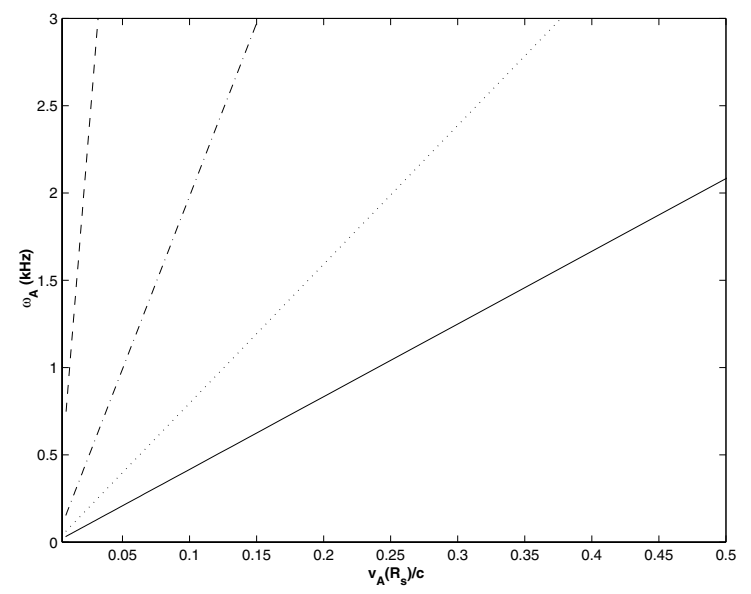

Fig. 4. Frequency of the regular Alfvén resonance $\left(v_{\mathrm{p}}=0\right)$ as a function of the Alfvén wave speed at the stellar radius, $\omega_{\mathrm{A}}=$ $k_{z} v_{\mathrm{A}}\left(R_{\mathrm{S}}\right)\left(r / R_{\mathrm{S}}\right)^{-9 / 4}$. The larger the Alfvén speed the higher the frequency. From top to bottom $r / R_{\mathrm{s}}=1,2,3$, and 4, respectively. Here $R_{\mathrm{s}}=10 \mathrm{~km}$, and $k_{z}=\pi / R_{\mathrm{s}}$.

Alfvén velocities at the surface of the $\operatorname{star}^{8}$. Here $\mu_{26}$ is the magnetic field dipole moment at the surface of the star in units of $10^{26} \mathrm{G} \mathrm{cm}^{3}, \dot{M}_{17}$ is the mass of accretion rate in units of $10^{17} \mathrm{~g} \mathrm{~s}^{-1}, x=r / R_{\mathrm{s}}$, and $R_{\mathrm{s}}$ is the radius of the star. Figures 3 and 4 show the regular Alfvén resonance frequencies $\left(v_{\mathrm{p}}=0\right)$

\footnotetext{
8 To properly calculate the Alfvén velocity at the surface of the star, one would need to use the relativistic Alfvén velocity as defined in Eq. (19), i.e.

$v_{\mathrm{A}}(r)=\frac{\mu \dot{M}^{-1 / 2}(2 G M)^{1 / 4} r^{-9 / 4}}{\sqrt{1+\mu^{2} \dot{M}^{-1}(2 G M)^{1 / 2} r^{-9 / 2} / c^{2}}}$

Here we assumed $p=0$. Using the above relation the Alfvén velocity at the surface of the star will be $\sim 0.8 c, .997 c$, and $c$ for $\mu=$ $10^{26}, 10^{27}$, and $10^{28} \mathrm{G} \mathrm{cm}^{3}$, respectively. At further distances from the star, however, the relativistic Alfvén velocity reduces to its classical version which we used instead. The values given here for the relativistic Alfvén velocity at the stellar surface are for a neutron star with $R_{\mathrm{S}}=10 \mathrm{~km}, \dot{M}=10^{17} \mathrm{~g} \mathrm{~s}^{-1}$ and $M=M_{\odot}$.
}

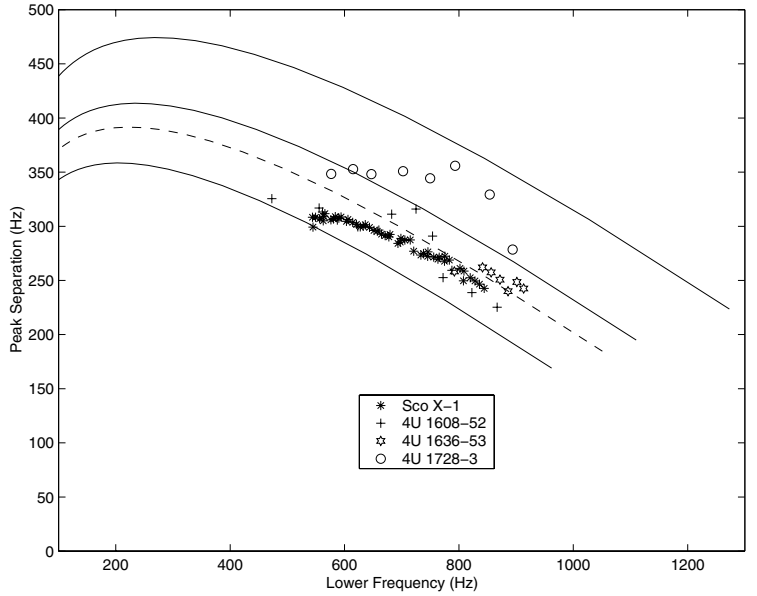

Fig. 5. Variation of the spectral peak separation $\Delta v$ with the lower frequency $v_{1}$, see Eqs. (31a) and (31c). The resulting curves are compared with the observed values for different systems. The solid curves from bottom to top correspond to $k_{z} v_{\mathrm{p}}\left(R_{\mathrm{s}}\right) \sim 650,750$ and $860 \mathrm{~Hz}$. The dashed curve corresponds to $k_{z} v_{\mathrm{p}}\left(R_{\mathrm{s}}\right) \sim 710 \mathrm{~Hz}$. Here we assumed $\mu_{26}=.32, R_{\mathrm{s}}=10 \mathrm{~km}, \dot{M}_{17}=1$, and $M=M_{\odot}$.

or FLRs, as a function of distance from the star and the Alfvén speed. It is clear that the closer to the star and/or the larger the Alfvén velocity the higher the frequency. Therefore, for an Alfvén speed of say $v_{\mathrm{A}}\left(R_{\mathrm{S}}\right) \sim .1 c$ one obtains the frequency of about $1000 \mathrm{~Hz}$ at $r \simeq 2.78 R_{\mathrm{s}}$ as expected. Furthermore, since the Alfvén velocity depends on the energy density (or density) of the plasma which varies due to several processes such as magneto-turbulence at boundaries during the accretion, the resulting FLR frequencies will also vary with time. In addition, the position where the resonance takes place is also subject to change in time due to the time-varying accretion rate. Such time-varying behavior causes time varying frequencies or quasi-periodic frequencies, as observed.

Setting $\omega^{-}$as the observed peak separation frequency $\Delta v \sim$ $\omega^{-}$and $\omega^{+}$as the upper QPO frequency $v_{2} \sim \omega^{+}$, one can find $v_{1}, v_{2}$ and $\Delta v$ as

$$
\begin{aligned}
v_{1}(x) \simeq 5 k_{z} v_{\mathrm{p}}\left(R_{\mathrm{S}}\right) \mu_{26} \dot{M}_{17}^{-1 / 2}\left(M / M_{\odot}\right)^{-1 / 4} & \\
& \times\left(R_{\mathrm{S}} / 10 \mathrm{~km}\right)^{-7 / 4} x^{-9 / 4} \\
v_{2}(x) \simeq k_{z} v_{\mathrm{p}}\left(R_{\mathrm{S}}\right)\left[x^{-1 / 2}+\right. & 2.5 \mu_{26} \dot{M}_{17}^{-1 / 2}\left(M / M_{\odot}\right)^{-1 / 4} \\
\times & \left.\left(R_{\mathrm{s}} / 10 \mathrm{~km}\right)^{-7 / 4} x^{-9 / 4}\right] \\
\Delta v(x) \simeq k_{z} v_{\mathrm{p}}\left(R_{\mathrm{S}}\right)\left[x^{-1 / 2}-\right. & 2.5 \mu_{26} \dot{M}_{17}^{-1 / 2}\left(M / M_{\odot}\right)^{-1 / 4} \\
\times & \left.\left(R_{\mathrm{s}} / 10 \mathrm{~km}\right)^{-7 / 4} x^{-9 / 4}\right]
\end{aligned}
$$

Figures 5 and 6 show the variation of $\Delta v$ vs. $v_{1}$ and $v_{2}$, respectively, along with the observed values. The curves are drawn by assuming $\mu_{26}=.32$ with (top to bottom) $k_{z} v_{\mathrm{p}}\left(R_{\mathrm{s}}\right) \sim 860 \mathrm{~Hz}$, $750 \mathrm{~Hz}$, and $650 \mathrm{~Hz}$. As shown by observations, the value of $\Delta v$ decreases when the magnitude of $v_{2}$ decreases (sufficiently) and/or it increases. Such behavior is expected in our model (see Fig. 6). 


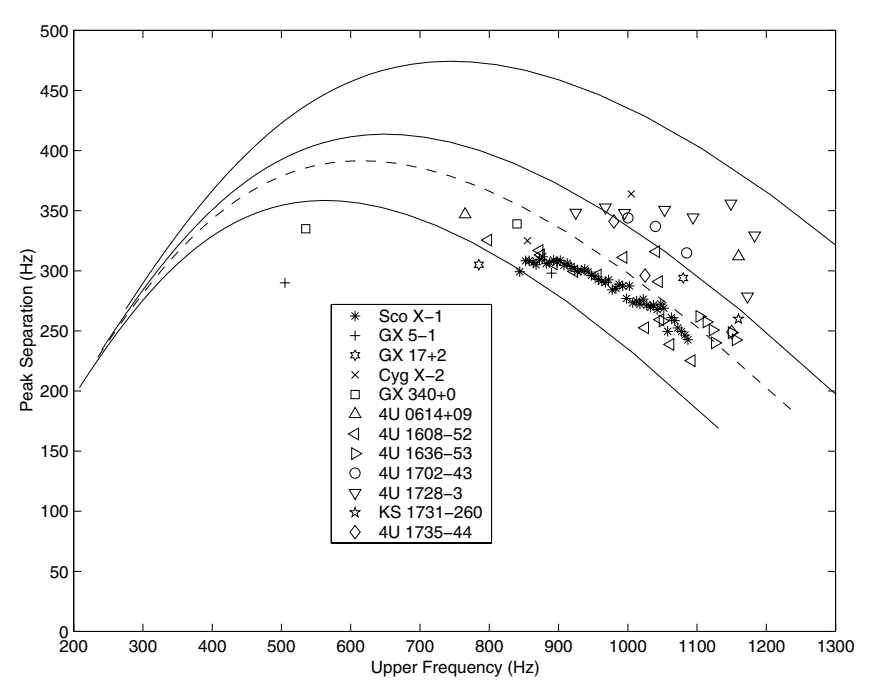

Fig. 6. Variation of the spectral peak separation $\Delta v$ with the upper frequency $v_{2}$, see Eqs. (31b) and (31c). The resulting curves are compared with the observed values for different systems. The solid curves from bottom to top correspond to $k_{z} v_{\mathrm{p}}\left(R_{\mathrm{S}}\right) \sim 650,750$ and $860 \mathrm{~Hz}$. The dashed curve corresponds to $k_{z} v_{\mathrm{p}}\left(R_{\mathrm{s}}\right) \sim 710 \mathrm{~Hz}$. Here we assumed $\mu_{26}=.32, R_{\mathrm{s}}=10 \mathrm{~km}, \dot{M}_{17}=1$, and $M=M_{\odot}$.

The lower QPO frequency can also be rewritten as $v_{1}=$ $v_{2}-\Delta v \sim 2 k_{z} v_{\mathrm{A}}$. As a result, we obtain the frequency ratio $v_{1}$ to $v_{2}$

$v_{1} / v_{2}=2 v_{\mathrm{A}} /\left(v_{\mathrm{p}}+v_{\mathrm{A}}\right)=2 /\left(1+v_{\mathrm{p}} / v_{\mathrm{A}}\right)$.

It is clear that depending on the relation between the plasma flow velocity $v_{\mathrm{p}}$ and the Alfvén velocity $v_{\mathrm{A}}$, one can obtain different frequency ratios. For example, if the velocity of the inflow plasma is $2(7 / 3 \sim 2.3)$ times greater than the Alfvén velocity, i.e. $v_{\mathrm{p}}=2 v_{\mathrm{A}}\left(v_{\mathrm{p}}=7 / 3 v_{\mathrm{A}}\right)$, one can get a 2:3 (3:5) ratio. For a free-falling plasma along the lines of a dipolar magnetic field, $v_{\mathrm{p}}(r) \propto r^{-1 / 2}$, whereas $B(r) / \sqrt{4 \pi \rho(r)} \propto r^{-9 / 4}$, the condition $v_{\mathrm{p}} \sim 2 v_{\mathrm{A}}\left(v_{\mathrm{p}} \sim 2.3 v_{\mathrm{A}}\right)$ is satisfied at $r \sim 3 R_{\mathrm{S}} \sim 1.5 r_{\mathrm{A}}$ (here $\mu_{26}=1$ is assumed). The occurrence of FLRs is very likely to be at this distance from the star.

Such pairs of high frequencies with a 2:3 frequency ratio have been observed in the X-ray flux of some neutron stars in LMXBs $^{9}$ and black hole systems (the 3:5 ratio has been in one source), and it would appear that this feature is common to those systems. However, recent statistical analyses by Belloni et al. $(2004,2005)$ showed that although the two high and low kHz QPO frequencies in LMXBs are well correlated, the frequency-frequency correlation is significantly different from a 2:3 relation. They analyzed all published values of $\mathrm{kHz}$ QPO frequencies from neutron star systems and found that the strong linear correlation observed between the lower and upper $\mathrm{kHz}$ QPO frequencies in both Atoll and Z sources is not compatible with a single constant ratio. Furthermore, by analysis of a much larger RXTE/PCA dataset for Sco X-1, they showed that there is no sharp concentration around a

${ }^{9}$ In Sco X-1 the correlation line between two frequencies has a steeper slope than 2/3 (see Belloni et al. 2004).
$2: 3$ ratio, but that the ratios are broadly distributed over the range $820-1150 \mathrm{~Hz}$.

The explanation for such rational ratios is still a mystery. In black hole systems, it has been suggested that such frequencies correspond to a trapped g-mode or c-mode of disk oscillation in the Kerr metric, see for example Kato (2001) and references therein. In neutron star systems they are explained as the fundamental and the first harmonic of the non-axisymmetric ( $m=1)$ g-mode (Kato 2002, 2003). Further, Rezzolla et al. (2003a,b) studied small perturbations of an accretion torus orbiting close to the black hole and modeled the observed high QPO frequencies with basic p-modes of relativistic tori. They showed that these modes behave as sound waves trapped in the torus with eigenfrequencies appearing in the sequence $2: 3: 4: \ldots$ Abramowicz et al. (2003) also proposed that the observed rational ratios of frequencies may be due to the strong gravity of the compact object and a non-linear resonance between radial and vertical oscillations in accretion disks.

\section{Discussion}

In the present work, we have studied the interaction of an accretion disk with a neutron star magnetosphere in the LMXBs. The recent extensive observations reveal the existence of quasiperiodic oscillations in the X-ray fluxes of such stars. These oscillations, with frequencies ranging from $10 \mathrm{~Hz}$ to $1200 \mathrm{~Hz}$, have been the subject of several theoretical and observational investigations. Based on theoretical models for the observed aurora in the Earth's magnetosphere, we have introduced a generic magnetospheric model for accretion disk-neutron star systems to address the occurrence and the behavior of the observed QPOs in those systems. In order to explain those QPOs consistently, we consider the interaction of the accreting plasma with the neutron star magnetosphere. Due to the strong gravity of the star, a very steep and supersonic flow hits the magnetosphere boundary and deforms its structure drastically. Such a plasma flow can readily excite different MHD waves in the magnetosphere, including shear Alfvén waves.

In the Earth's magnetosphere, occurrence of aurorae is a result of the resonant coupling between the shear Alfvén waves and the fast compressional waves (produced by the solar wind). These resonances are known as FLRs and are reviewed in detail in Sect. 2 of this paper. We argue that such resonant coupling is likely to occur in neutron star magnetospheres due to the interaction with accreting plasma. In the context of QPOs in neutron star magnetospheres, we formulated an improved FLR model by considering a plasma flow moving with velocity $v_{\mathrm{p}}$ along the magnetic field lines. Such flows are likely to occur in a neutron star magnetosphere (Ghosh et al. 1977). For a simple geometry, a rectilinear magnetic field, and in the presence of a plasma flow we found: (a) two resonant MHD modes with frequencies $\omega^{ \pm}=k_{z}\left(v_{\mathrm{p}} \pm v_{\mathrm{A}}\right)$; (b) the resulting frequencies for $k_{z} \sim \pi / R_{\mathrm{s}} \sim 3 \times 10^{-6} \mathrm{~cm}^{-1}$ and typical flow and/or Alfvén velocity $\sim .1 c$ at the stellar surface will be in $\mathrm{kHz}$ range within a few stellar radii. Our results match the $\mathrm{kHz}$ oscillations observed in the X-ray fluxes in LMXBs. As shown in Figs. 3 and 4, the closer to the star and/or the larger the Alfvén velocity the higher the frequency; (c) the quasi-periodicity of 
the observed oscillations can be understood by noting that due to several processes such as magneto-turbulence at boundaries and the time varying accretion rate, the FLR frequencies may vary with time; (d) a non-zero plasma displacement along the magnetic field lines $\xi_{\|}$. Such a displacement, which oscillates with resulting frequencies $\omega^{ \pm}$, modulates the flow of the plasma toward the surface of the neutron star. As a result, the X-ray flux from the star will show these frequencies as well; (e) setting $\Delta v=\omega^{-}$and $v_{2}=\omega^{+}$, one can explain the behavior of the peak separation frequency $\Delta v$ relative to the upper QPO frequency $v_{2}$. As observed, the value of $\Delta v$ decreases as the magnitude of $v_{2}$ decreases and/or increases. Figure 6 clearly shows such a behavior; (f) for $v_{\mathrm{p}} \sim 2 v_{\mathrm{A}}$ (obtained at $r \sim 3 R_{\mathrm{S}}$ for $\mu_{26} \sim 1$ ), the frequency ratio $v_{1} / v_{2}$ is comparable with the observed frequency ratio $2: 3$ in some neutron star systems. However, a broad range of frequency ratios (Belloni et al. 2004, 2005 ) is expected as values of $v_{\mathrm{p}}$ and $v_{\mathrm{A}}$ change.

Interestingly, using the observed values of QPO frequencies, one can determine the mass density and the magnetic dipole moment of the star using

$k_{z} v_{\mathrm{p}}(r)=(1 / 2)\left(v_{2}+\Delta v\right)=v_{2}-v_{1} / 2$,

$k_{z} v_{\mathrm{A}}(r)=(1 / 2)\left(v_{2}-\Delta v\right)=v_{1} / 2$.

Therefore,

$M_{\mathrm{s}} / R_{\mathrm{s}}^{3} \simeq \frac{1}{8 \pi^{2} G} x^{3}\left(v_{2}+\Delta v\right)^{2}$,

$\mu_{\mathrm{s}} \simeq\left(32 \pi^{4} G\right)^{-1 / 4} R_{\mathrm{s}}^{5 / 2} \dot{M}^{1 / 2}\left(M_{\mathrm{s}} / R_{\mathrm{s}}^{3}\right)^{-1 / 4} x^{13 / 4} v_{1}$,

where $k_{z} \simeq \pi / r, \mu_{\mathrm{s}}=B_{0} R_{\mathrm{s}}^{3}, x=r / R_{\mathrm{s}}$, and $B_{0}$ is the magnetic field strength at the surface of the star. In Table 1, we calculate the average density $M_{\mathrm{s}} / R_{\mathrm{s}}^{3}$ and magnetic dipole moment $\mu_{\mathrm{s}}$ of the star for a fixed value $x=10$. Note that larger frequencies may occur at smaller distances, i.e. $x<10$. Our results are compatible with realistic neutron star parameters.

Furthermore, our model is able to explain the low frequency $(\sim 10 \mathrm{~Hz})$ quasi-periodic oscillations observed in rapid bursters such as MXB 1730-335 and GRO J1744-28 (Masetti et al. 2000). Such low frequencies can be extracted from the model by considering smaller inflow/Alfvén velocities and/or further distances from the star.

Nevertheless, in order to avoid a number of complexities in our calculations, we used approximations such as slow rotation and a cold plasma. These assumptions may place some restrictions on the validity of our model and results. Future studies will be devoted to overcoming these restrictions.

Acknowledgements. V.R. wishes to thank Mariano Mendez for kindly providing QPO data. The authors appreciate the referee's careful reading of the manuscript and valuable suggestions. This research was supported by the National Sciences and Engineering Research Council of Canada.

\section{References}

Abramowicz, M. A., Karas, V., Kluzniak, W., Lee, W. H., \& Rebusco, P. 2003, PASJ, 55, 467

Alpar, M. A., \& Shaham, J. 1985, Nature, 316, 239

Belloni, T., Mendez, M., \& Homan, J. 2004, Proc. the NATO/ASI: The Elecromagnetic Spectrum of Neutron Stars, June 7-18 2004 (Turkey: Marmaris), ed. A. Baykal, S. K. Yerli, M. Gilfanov, \& S. Grebenev, in press

Belloni, T., Mendez, M., \& Homan, J. 2005, A\&A, in press [arXiv: astro-ph/0501186]

Chakrabarty, D., Morgan, E. H., Muno, M. P., et al. 2003, Nature, 424, 42

Chen, L., \& Hasegawa, A. 1974, J. Geophys. Res., 79, 1024

Elsner, R. F., \& Lamb, F. K. 1977, ApJ, 215, 897

Elsner, R. F., \& Lamb, F. K. 1984, ApJ, 278, 326

Ghosh, P., Lamb, F. K., \& Pethick, C. J. 1977, ApJ, 217, 578

Harrold, B. G., \& Samson, J. C. 1992, Geoph. Res. Let., 19, 1811

Hasegawa, A. 1976, J. Geophys. Res., 81, 5083

Kato, S. 2001, PASJ, 53, 1

Kato, S. 2002, PASJ, 54, 39

Kato, S. 2003, PASJ, 55, 801

Lamb, F. K., \& Miller, M. C. 2001, ApJ, 554, 1210

Lamb, F. K., Shibazaki, N., Alpar, M. A., \& Shaham, J. 1985, Nature, 317,681

Landau, L. D., \& Lifshitz, E. M. 1992, Elektrodinamika Splosnych Sred (Moscow: Nauka)

Liu, W. W. 1997, J. Geophys. Res., 102, 4927

Masetti, N., Frontera, F., Stella, L., et al. 2000, A\&A, 363, 188

Mestel, L. 1968a, MNRAS, 138, 359

Mestel, L. 1968b, MNRAS, 140, 177

Middleditch, J., \& Priedhorsky, W. C. 1986, ApJ, 306, 230

Miller, M. C., Lamb, F. K., \& Psaltis, D. 1996, American Astronomical Society, 189th A\&AS Meeting, \# 44.11; BAAS, 28, 1329

Miller, M. C., Lamb, F. K., \& Psaltis, D. 1998, ApJ, 508, 791

Mizuno, Y., Yamada, S., Koide, S., \& Shibata, K. 2004, ApJ, 606, 395

Ohtani, S.-I., \& Tamao, T. 1993, J. Geophys. Res., 98, 19369

Rezzolla, L., Yoshida, S., Maccarone, T. J., \& Zanotti, O. 2003a, MNRAS, 344, L37

Rezzolla, L., Yoshida, S., \& Zanotti, O. 2003b, MNRAS, 344, 978

Rickard, G. J., \& Wright, A. N. 1994, J. Geophys. Res., 99, 13455

Samson, J. C. 1991, in Geomagnetism, ed. J. A. Jacobs (Academic press), 4, 482

Samson, J. C., Rankin, R., \& Tikhonchuk, V. T. 2003, Ann. Geophys., 21, 933

Southwood, D. J. 1974, Planet. Space Sci., 22, 483

Stella, L., \& Vietri, M. 1998, ApJ, 492, L59

Stella, L., \& Vietri, M. 1999, Phys. Rev. Lett., 82, 17

Stix, T. H. 1992, Waves in Plasma (New York: American Institute of Physics Press)

Taylor, J. P. H., \& Walker, A. D. M. 1984, Planet. Space Sci., 32, 1119 van der Klis, M., Jansen, F., van Paradijs, J., et al. 1985, Nature, 316, 225

van der Klis, M. 2000, ARA\&A, 38, 717

Wijnands, R., van der Klis, M., Homan, J., et al. 2003, Nature, 424, 44

Zhang, C. 2004, A\&A, 423, 401 